\title{
The Fine Structure of Differentiating Fibroblasts in the Incisor Pulp of the Guinea Pig '
}

\author{
SEONG S. HAN, JAMES K. AVERY AND LAWRENCE E. HALE \\ The University of Michigan School of Dentistry and \\ Department of Anatomy, Ann Arbor, Michigan
}

\begin{abstract}
The differentiation of the fibroblast was followed in the dental pulp of continuously growing incisors of the guinea pig. Based on the ultrastructure, the processes of differentiation of the fibroblasts might be conveniently broken into three stages; Stage I - period of early differentiation, Stage II - period of maturation and functioning, and Stage III - period of regression.

During Stage I the cell had structural characteristics shared by other less differentiated cells. The endoplasmic reticulum was poorly developed, showing vesicular to tubular profiles. The ribosomes were abundant but were mostly distributed in free form. Mitochondria were small and had irregular interiors.

Stage II was characterized by a striking development of the rough-surfaced endoplasmic reticulum, which appeared in various shapes and sizes. The Golgi complex was enlarged, and contained some fibrillar materials in dilated portions of its membraneous elements. Other features described in the cytoplasm of fibroblasts from other sources were confirmed.

Stage III was characterized by the decrease in size and number of various cytoplasmic constituents and was considered to represent cells in the state of regression.
\end{abstract}

The fine structure of the fibroblast has been characterized by many previous workers (Ref. reviews by Chapman, '62; and Porter, '64) and we have described the cell and related structures in the dental pulp (Avery and Han, '61; and Han and Avery, '63). However, only incidental information has been made available in terms of the structural characteristic of the cell during its normal differentiation.

The dental pulp of continuously growing rodent incisors provides us with an unusually favorable material for study of the fine structure of differentiating fibroblasts (fig. 1). At the proximal or growing end (A) are located numerous primitive cells that differentiate into pulpal fibroblasts as they migrate to the mid-pulp region (B). At the distal or incisal end (C) are seen more mature fibroblasts and fibrocytes than at the former regions. The present article is aimed at describing the ultrastructural aspects of differentiating fibroblasts as observed in the dental pulp of guinea pig incisors.

\section{MATERIALS AND METHODS}

Twenty guinea pigs, each weighing approximately $250 \mathrm{gm}$, were used in this study. While the animals were under ether anesthesia mandibles were rapidly dissected out and bisected along the midline. The body of the mandible was clipped off with shears along the lateral aspects of the base from the angle forward and the entire incisor, including the growing end, was exposed. Then the tooth was separated from the mandible and, following a brief dip in the fixative, the partially calcified dentin from the proximal portion was removed. The pulp thus exposed was removed from the hard shell of dentin by gently pulling with forceps.

Form different regions of the pulp (fig. 1) small pieces of the tissue were cut and fixed either in $6.5 \%$ glutaraldehyde or in $2 \%$ osmic acid, both of which were buffered with $\mathrm{M} / 10$ phosphate at $\mathrm{pH}$ 7.4. Tissues that had been fixed with glutaraldehyde were washed briefly in the buffer and refixed in $2 \%$ osmic acid. Sucrose was added to the osmic acid to make a concentration of $4.5 \%$ sucrose. Following fixation the tissues were dehydrated through a graduated series of ethanol and embedded in a mixture of epoxy resin. Sections were cut on a Porter-Blum or LKB ultramicrotome and picked up on formvar-

\footnotetext{
1 Supported in part by grant D-1620 from The United States Public Health Service.
} 


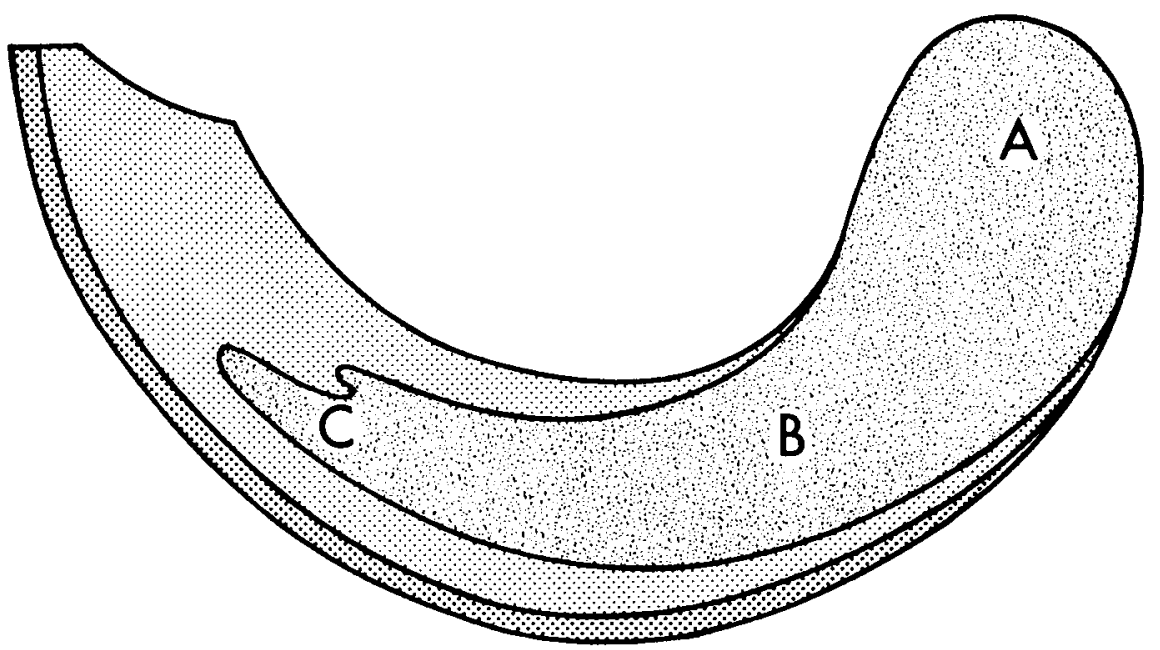

Fig. 1 A diagram of the sagittal view of a mandible incisor of the guinea pig. The fine stippling indicates the position of the pulp. Cells from the growing end (A) are primitive, whereas cells from the mid-pulp (B) have structural characteristics of the fully mature fibroblast, and fibrocytes from the incisal end (C) show the regressive changes described in the text.

coated grids reinforced with a thin layer of carbon. Saturated uranyl acetate and $0.1 \%$ phosphotungstic acid solutions were used as electron stains. Observations were made in a Hitachi HU-11 electron microscope.

\section{OBSERVATIONS}

Based on the fine structure, the description of differentiation of the fibroblast may be conveniently broken into three arbitrary stages: Stage I, period of early differentiation; Stage II, period of maturation and functioning; and Stage III, period of regression. Table 1 is a summary of structural characteristics of the cell during these stages.

\section{Stage I Early differentiation}

Most cells present in the growing end of the incisor pulp had intracellular structures which might be characteristic of other primitive cells (figs. 3 and 4 ). The cell body was of polygonal shape and contained a number of vesicles that appeared to be elements of the smooth-surfaced endoplasmic reticulum. Occasionally profiles of the rough-surfaced endoplasmic reticulum (RER) were noted. Many of them had a tubular appearance. In some cells where increased numbers of the RER were found, coalescence of small vesicles with the RER was often seen (fig. 4). Although a variable number of ribosomes was seen, most of them were scattered freely throughout the cytoplasm. The Golgi complex was small and inconspicuous. Usually a few short bilaminar components stacked up to form a recognizable Golgi element. One or two of such stacks with only a small number of associated vesicles were located among other organelles (fig. 3).

Mitochondria were small and variable in number, being more numerous in cells with greater numbers of ribosomes, and were mostly of oval to elongate shapes. Cristae mitochondriales were few in number and often irregular in shape, transversing the matrix in different directions. The mitochondrial matrix was moderately electron-dense. In cells which contained an increased number of the RER, mitochondria with an elongated shape were found more frequently (fig. 4). However, their cristae were also irregular. The nucleus was usually round, and had a smooth nuclear envelope. A varying number of ribosomes was located on its outer surface. Nucleoli, when present, were large and located at the periphery (fig. 3 ). 
DIFFERENTIATION OF FIBROBLASTS
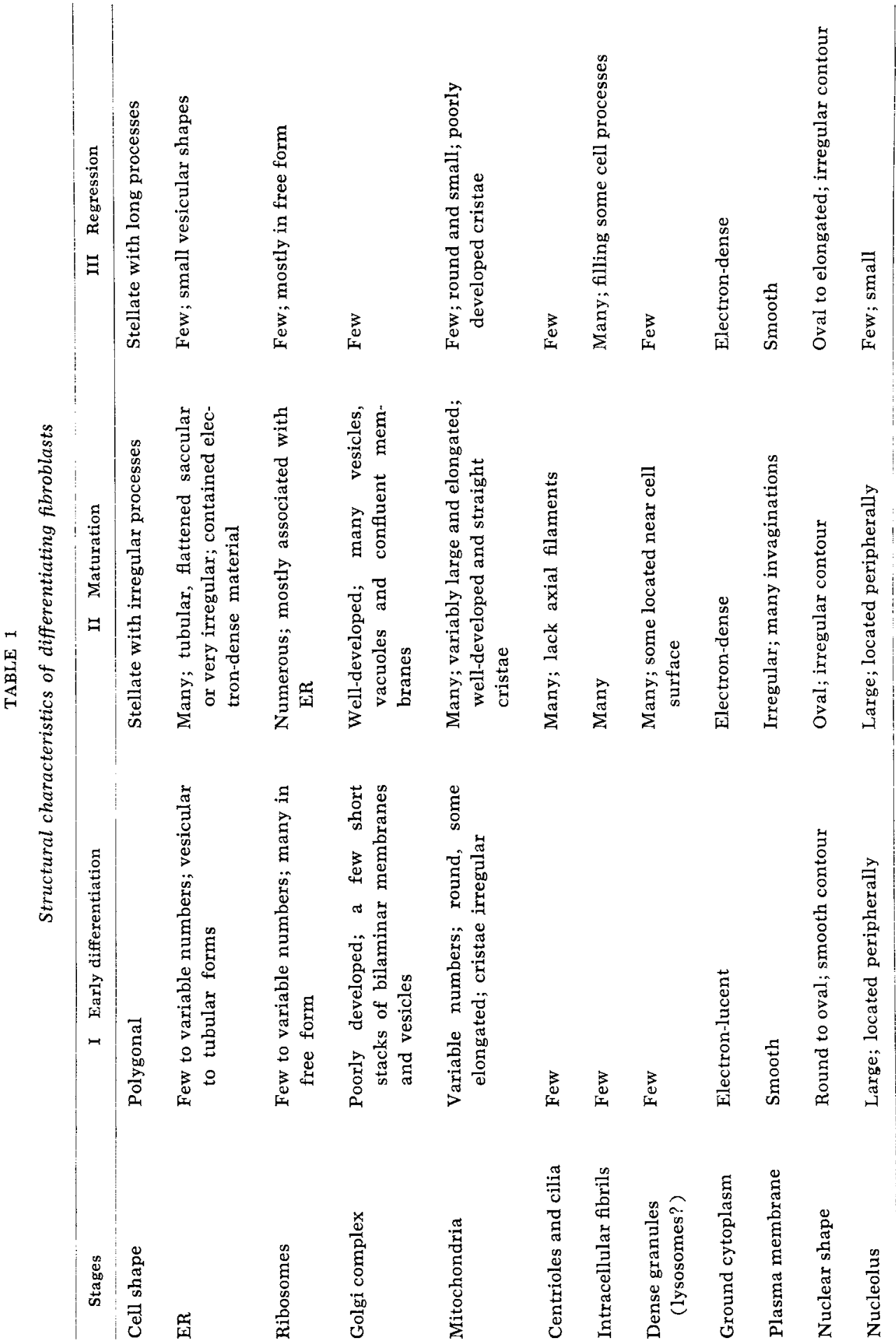


\section{Stage II Period of maturation and functioning}

Well developed intracellular organelles with increased complexity in structure characterized this stage. Their appearance suggested that the cell had achieved a high degree of maturation and was functioning as a fibroblast. Cells of this stage, most frequently found in the midportion of the pulp, were stellate in shape and had long cytoplasmic extensions (fig. 2).

Changes in structure of the intracellular organelles. As in fibroblasts found in other connective tissues, the RER was very prominent and appeared as tubular to saccular profiles (figs. 2, 5, 6, 7 and 8). Cisternae of the RER were extremely dilated in some cells (fig. 8). In sections such dilations appeared as lakes of irregular outline with islets of the cytoplasm within them. The interior of the dilated RER contained flocculent materials which had in electron density similar to that of extracellular substances, whereas the RER which appeared as tight tubules and flattened sacs contained intracisternal materials which much denser than that of extracellular substances (figs. 5 and 7 ).

The Golgi complex was markedly enlarged and occupied a juxtanuclear location demonstrating an extensively developed stack of bilaminar membranes and a large number of vesicles and vacuoles (fig. 6). Continuity of vesicles with RER as well as with membranes of the Golgi complex has been observed (fig. 6). Occasionally dense fibrillar materials were seen to be enclosed by vesicular and vacuolar membranes which appeared to belong to the Golgi complex. Mitochondria were elongated, increased in number and large in size which varied considerably from cell to cell. Cristae mitochondriales were numerous and more straight than in primitive cells, and ran transversely across the mitochondrial matrix.

The ground cytoplasm had become dense and contained varying numbers of intracellular fibrils measuring about $50 \AA$ in diameter (figs. 2, 5, 8, 9 and 14). They were less obvious in cells with the RER of flat profiles, whereas in cells with dilated RER they were seen more clearly. Occasionally these fibrils formed a bundle or tract which was located within the marginal cytoplasm in close association with the plasma membrane (fig. 8). Scattered between the intracellular fibrils were a moderate number of ribosomes. In many instances several ribosomes aggregated to form a cluster (figs. 2 and 9). Elsewhere in the cytoplasm a small number of granules enclosed by a membrane were present (figs. 2, 7, 9, 13 and 14). Such granules were extremely dense and their average diameter measured about $0.5 \mu$. They appeared throughout the cytoplasm, often close to the plasma membrane.

A cilium was frequently found near the nucleus (figs. 17 and 18). Occasionally a centriole was located perpendicular to the long axis of the cilium. Almost always a Golgi complex, encompassing a small amount of the cytoplasm which contained the cilium and/or centriole, was seen in the region. This portion of cytoplasm surrounded by the Golgi complex was characteristically free of organelles except for a few aggregates of ribosomes. The cilium consisted of a basal body, ciliary shaft and sheath. As in other non-motile cilia found elsewhere, the central tubules of the basal body were not evident. Ofter a couple of rootlets originated from the distal end of the basal body and extended for a short distance at an angle. The shaft was continuous with the basal body, and the sheath formed deep invaginations at its proximal end, demonstrating, when sectioned transversely, a circular to semicirular areas of extracellular space around the shaft (figs. 2 and 5). The junction of the sheath and plasma membrane in the invaginated region has a small amount of subjacent cytoplasm which was notably dense.

The nucleus of the fibroblast was oval to elongated. The nuclear membrane was more irregular than in Stage I and exhibited a jagged contour (figs. 5 and 14). When the nuclear contour was relatively smooth it often had a deep notch (fig. 2). One or more large nucleoli were located at the periphery.

Appearance of surface plasma membranes. Along the surface of the cell a number of small invaginations, vesicles and vacuoles suggesting pinocytic activities were present (figs. 2, 7 and 9). They ap- 
peared most frequently in cells having flat profiles of the RER which contained an electron dense intracisternal material. The inner surface of these vesicles was covered by a thin layer of electron dense materials which seemed to be continuous to similar materials along the surface of the plasma membrane.

On the other hand, the surface plasma membrane of cells with dilated RER had portions that were exteremely irregular (figs. 10 and 11). Deep invaginations as well as cytoplasmic protrusions of various sizes were observed. The appearance of such regions often suggested that the dilated ER and the plasma membrane might come in contact with resulting confluence of the two (fig. 11). This could cause the irregularity of the plasma membrane and the release of intracisternal materials to the extracellular space. In addition, the appearance of small protrusions and islands of cytoplasm away from the surface suggested that a separation from the cell of bits of cytoplasm in this region could be possible. Many of the cytoplasmic bits so separated contained a large number of ribosome-like granules which did not cluster as was seen in the cell (fig. 10).

\section{Stage III Period of regression}

As fibroblasts approached the distal end of the pulp they had less perinuclear cytoplasm than was observed in fibroblasts in more proximal regions (figs. 15 and 16). Although peripheral processes were also thinner than in cells described previously, they were still very long. Most intracellular organelles became small and showed signs of regression. The RER was greatly reduced in size and in number. Several vesicular profiles were seen in the perinuclear region. Only a nominal number of ribosomes was present in small clusters. The Golgi complex was seldom observed. Mitochondria were also decreased in number and size. They usually were round and showed a few inconspicuous cristae.

Elsewhere in the ground cytoplasm the fine intracellular fibrils appeared to remain relatively well preserved in comparison to other organelles. In peripheral processes bundles of these fibrils were arranged in definite directions, so that the intracellular fibrils of two adjacent cell processes might run at almost right angles to each other (fig. 16). In addition, small vesicles were present in varying numbers. Whether they represented the smooth-surfaced endoplasmic reticulum or results of pinocytosis was not clear. The rest of the ground cytoplasm was fairly dense.

The nucleus almost filled the small cell body. The contour of nuclear membranes was quite irregular. Few nucleoli were observed.

\section{DISCUSSION}

Structure of the cells in primitive state and early differentiation. While there have been numerous reports describing the fine structure of fibroblasts and other collagen producing cells, (Ref. reviews by Chapman, '62; and Porter, '64) few have characterized the ultrastructural changes occurring during normal differentiation of the fibroblast from a primitive cell. Perhaps the diversity in functional status of the fibroblasts from usual sources might have presented difficulties in obtaining suitable materials which would permit such a study. On the other hand, our past experiences have shown that the process of differentiation of the fibroblasts could be successfully observed by taking advantage of the progressive differentiation of the fibroblast population in the dental pulp of continuously growing rodent incisors (fig. 1).

The fine structure of undifferentiated cells from different tissues and organs has been studied by various workers (Howatson and Ham, '55; Hay, '58; Fawcett, '59; Godman and Porter, '60; Karrer, '60; Han, '61; and others). The lack of extensive development of the RER, Golgi apparatus and intracellular fibrils has been consistently observed in most immature cells and has been confirmed in the present study. The ground cytoplasm was electron-lucent, supporting the contention that the primitive cells are more hydrated than mature ones (Eichelberg, '58).

With regards to the relative number of ribosomes and mitochondria among cells. the individual variation observed in this study might be taken to indicate the diverse physiological status of cells in this rapidly growing portion of the pulp. Since free ribosomes are considered to be responsible for the synthesis of proteins necessary for 
the growth of the cell itself, their number might be related to the growth rate of individual cells.

The importance of mitochondrial structure in terms of biochemical functioning has been ascertained repeatedly (Green and Hatefi, '61; Fernández-Morán, '62; and Stoeckenius, '63). In primitive cells, the intramitochondrial structure appears char acteristically irregular regardless of the rate of their growth. For instance, the irregularity in mitochondrial structure was observed in inactive primitive reticular cells of the lymph node, as well as in highly prolific hemocytoblasts of the spleen from immunologically challenged animals (Han, '61; and Han et al., '64), and also was a characteristic feature of immature cells reported in this study. Therefore, it might be possible that the increased demand for cellular energy in the early developmental stage is possibly met by the increase in number and in size of the mitochondria, whereas the differentiation of mitochondrial structure may reflect the acquisition of certain biochemical functions specific to the cell type. A mitochondrion of a muscle cell whose primary biochemical function is the production of adenosintriphosphate, for example, is readily distinguished from that of a liver cell which is concerned with a variety of intermediary metabolic activities (Porter and Bonneville, '64).

Mature fibroblasts. The increase in complexity and number of the RER, mitochondria and elements of the Golgi apparatus, as well as enhanced density of ground cytoplasm, indicates the attainment of full maturity of fibroblasts of the midpulp. Recent reviews have adequately presented the existing evidence on the basic cytoplasmic machinery of the fibroblast and its significance in relation to the synthesis of intercellular macromolecules (Chapman, '62; and Porter, '64) and therefore will not be discussed in detail here. It appears certain that the current consensus on the role of the RER and Golgi complex in the production of secretory proteins is also exemplified in the case of the fibroblast. Ross and Benditt ('62), in a radioautographic study of wound healing, have followed the movement of tritiated proline from the RERGolgi region to the extracellular space as the function of time. Based on observa- tions of the fibrillar structures in the Golgi apparatus of cartilage cells, Sheldon and Kimball ('62) suggested that, since the apparatus is believed to be concerned with concentration of secretory products, a favorable environment could be produced within the Golgi region for the registration of tropocollagen molecules to form regular collagen fibrils. The presence of fibrils observed in the Golgi region of mature fibroblasts of the dental pulp might be taken as another evidence for such a possibility. Furthermore, there is evidence that the Golgi apparatus might be concerned with the production of non-proteinic intercellular substances as judged by histochemical and radioautographic evidences (Peterson and Leblond, '64). If the ground substance promotes extracellularly the formation of collagen fibrils from tropocollagen molecules, then it is possible that the same may be effected in the Golgi apparatus where the ground substance is being formed.

Fawcett ('61) published a comprehensive review of the fine structure and functions of the cilia. Since then a number of observers have noted the presence of cilia in different types of cells (Barnes, '61; Latta et al., '61; Palay, '61; Taxi, '61; and others), including fibroblasts (Sorokin, '61). Some of them have the typical structure characteristics of motile cilia, whereas others are devoid of axial filaments. The ones found in fibroblasts of the dental pulp belong to the latter group, and whether this represents a rudimentary structure of cellular evolution or an organelle related to the sensory function as suggested by certain authors (Barnes, '61) cannot be determined, although, as pointed out by Grillo and Palay ('63), no sensory mechanisms are involved with most of those cells having atypical cilia.

Fibrocytes and regression. The quiescent nature of metabolism of fibrocytes located in the apical end of the pulp is well-reflected in the generalized reduction in size and/or number of cytoplasmic components which are metabolically active, namely the ribosomes, RER, Golgi complexes and mitochondria. The persistence of intracytoplasmic fibrils and of electrondense ground cytoplasm suggests that such a loss of active cytoplasmic structures may 
not represent a process of dedifferentiation but rather a simple regression of secretory functions as fibroblasts. In addition, the increased irregularity in the nuclear contour, and the small nucleolus of fibroblasts support the presumed reduction of nuclear activities. Porter ('64) claims that the number of nuclear pores is also reduced.

The increase in nucleocytoplasmic ratio of regressing cells could be explained on the basis of the following three possibilities. The first is that the electro-chemical organization of macromolecules filling the ground cytoplasm might be so changed that the degree of cytoplasmic hydration becomes reduced, resulting in the actual decrease of cell volume. The second is the possible release of parts of cytoplasm by a process of pinching off. The second possibility appears to be a common way employed by many types of cells in getting rid of parts of the cytoplasm for different reasons. For instance, the plasma cell release bits of cytoplasm during the course of secretion (Han et al., '64); the megakaryocyte forms and liberates the platelets by a pinching off of the peripheral cytoplasm (Yamada, '57; Han and Baker, '64); and the cultured epithelial cell, when injured with certain detergents, also eliminates parts of the cytoplasm by formation of little blebs which eventually become separated (Han and Avery, '63). The fragments of cytoplasm observed in the vicinity of fibroblasts of the midpulp region (figs. 10 and 11) might add to the examples described above and represent a situation analogous to antibody secretion by plasma cells. In fact, the phenomenon was observed earlier by Stearns (' $40 a, b$ ) in her study of connective tissue growth in transparent chambers placed in rabbit ears. The third is the possibility that there may not be an actual reduction in cell volume, but that the perinuclear cytoplasm is drawn into cell processes which might become more elongated as time goes on. In reality any one or combination of the three possibilities could account for the relative decrease of perinuclear cytoplasm.

\section{LITERATURE CITED}

Avery, J. K., and S. S. Han 1961 The formation of collagen fibrils in the dental pulp. J. Dent. Res., 40: 1248-1261.
Barnes, B. G. 1961 Ciliated secretory cells in the pars distalis of the mouse hypophysis. J. Ultrastr. Res., 5: 453-467.

Chapman, J. A. 1962 Fibroblasts and collagen. Brit. Med. Bull., 18: 233-237.

Eichelberger, L., W. H. Akeson and M. Roma 1958 Biochemical studies of articular cartilage. I. Normal values. J. Bone and Joint Surg., 40: 142-152.

Fawcett, D. W. 1959 Changes in the fine structure of the cytoplasmic organelles during differentiation. In: Developmental Cytology. D. Rudnick, ed., Ronald Press, New York, pp. 161189.

Fawcett, F. 1961 Cilia and flagella. In: The Cell, Vol. II. J. Brachet and A. E. Mirsky, ed., Acad. Press, New York and London, pp. 217297.

Fernández-Morán, H. 1962 Cell-membrane ultrastructure; low-temperature electron microscopy and $x$-ray diffraction studies of lipoprotein components in lamellar systems. Circulation, 26: 1039-1065.

Godman, G. C., and K. R. Porter 1960 Chondrogenesis, studied with electron microscope. J. Biophys. Biochem. Cytol., 8: 719-760.

Green, D. E., and Y. Hatefi 1961 The mitochondrion and biochemical machines. Science, 133: 13-19.

Grillo, M. A., and S. L. Palay 1963 Ciliated Schwann cells in the autonomic nervous system of the adult rat. J. Cell Biol., 16: 430-436.

Han, S. S. 1961 The ultrastructure of the mesenteric lymph node of the rat. Am. J. Anat., 109: 183-226.

Han, S. S., and J. K. Avery 1963 The ultrastructure of the connective tissue of the dental pulp. J. Dent. Res., 42: 95 (Abstract). 1963 Unpublished observations.

Han, S. S., and B. L. Baker 1964 The ultrastructure of megakaryocytes and blood platelets in the rat spleen. Anat. Rec., 149: 251-268.

Han, S. S., I. H. Han and A. G. Johnson 1964 The antibody response in the rat. II. Microelasmatosis, a possible mode of antibody release. Submitted for publication.

Hay, E. D. 1958 The fine structure of blastema cells and differentiating cartilage cells in regenerating limbs of amblystoma larvae. J. Biophys. Biochem. Cytol., 4: 583-592.

Howatson, A. F., and A. W. Ham 1955 Electron microscope study of sections of two rat liver tumors. Cancer Res., 15: 62-69.

Karrer, H. E. 1960 Electron microscope study of developing chick embryo aorta. J. Ultrastruct. Res., 4: 420-454.

Latta, H., A. B. Maunbach and S. C. Madden 1961 Cilia in different segments of the rat nephron. J. Biophys. Biochem. Cytol, 11: 248252.

Palay, S. L. 1961 Structural pecularities of the neurosecretory cells in the preotpic nucleus of the gold fish, Carassius Auratus. Anat. Rec., 139:262 (Abstract). 
Peterson, M. R., and C. P. Leblond 1964 Synthesis of complex carbohydrates in the Golgi zone, as shown by radioautography after injection of labeled glucose. J. Cell Biol., 21: 143-148.

Porter, K. R. 1964 Cell fine structure and biosynthesis of intercellular macromolecules. In: Connective Tissue: Intercellular Macromolecules. Proceedings of a symposium sponsored by the New York Heart Assoc. Little, Brown and Co., Boston, pp. 167-196.

Porter, K. R., and M. A. Bonnerville 1964 An Introduction to the Fine Structure of Cells and Tissues. Lea \& Febiger, Philadelphia.

Ross, R., and E. P. Benditt 1962 Wound healing and collagen formation. III. A quantitative radioautographic study of the utilization of proline $-\mathrm{H}^{3}$ in wounds from normal and scorbutic guinea pigs. J. Cell Biol., 15: 99108.

Sheldon, H., and F. B. Kimball 1962 Studies on cartilage. III. The occurrence of collagen within vacuoles of the Golgi apparatus. J. Cell Biol., 12: 599-614.

Sorokin, S. 1962 Centrioles and the formation of rudimentary cilia by fibroblasts and smooth muscle cells. J. Cell Biol., 15: 363-377.

Stearnes, M. L. $1940 \mathrm{a}$ Studies on the develop. ment of connective tissue in transparent chambers in the rabbit's ear. I. Am. J. Anat, 66: 133-176.

$1940 \mathrm{~b}$ Studies on the development ot connective tissue in transparent chambers in the rabbit's ear. II. Am. J. Anat, 67: 55-97. Stoeckenius, W. 1963 Some observations on negatively stained mitochondria. J. Cell Biol., 17: 443-454.

Taxi, J. 1961 Sur l'éxistence de neurones ciliés dans les ganglions sympathiques de certains Vertebrés. Comp. Rend. Soc. Biol., 160: 1860 . 1863.

Yamada, E. 1957 The fine structure of the megakaryocyte in the mouse spleen. Acta Anat., 29: 267-290.

PLATE 1

EXPLANATION OF FIGURE

2 A fibroblast from the middle portion of the incisor pulp of a guinea pig. The cell has a stellate shape and contains numerous well developed mitochondria, a fair number of flat profiles of RER, dense granules and intracellular fibrils. A ciliary shaft may be seen in the middle (arrow). The nucleus shows an indention, and has a large nucleolus. The cell might be in the early period of Stage II. Approximately $\times 17,000$. 


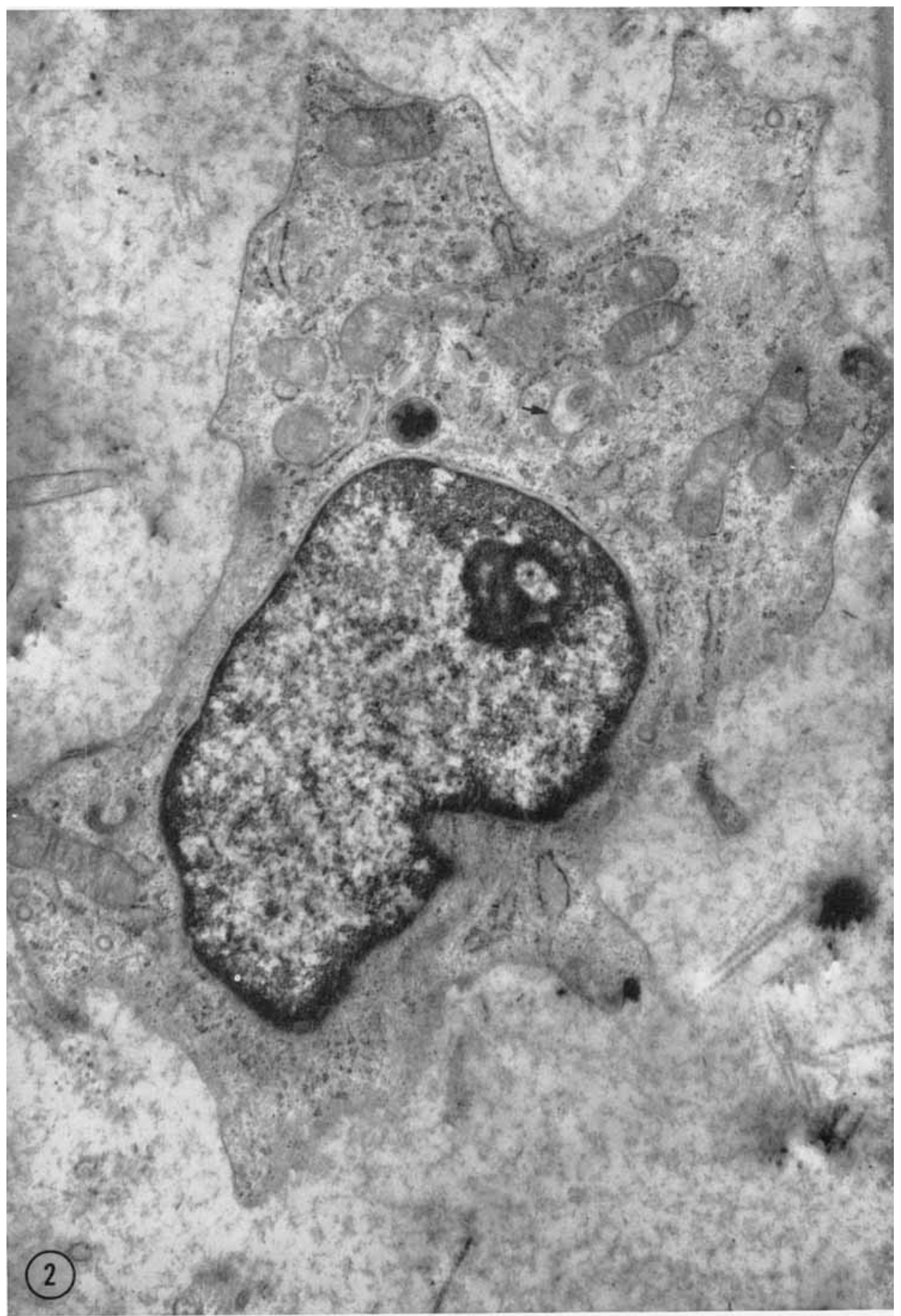




\section{PLATE 2}

EXPLANATION OF FIGURES

3 A portion of the cytoplasm of an immature cell from the growing end of a guinea pig incisor pulp. Note the numerous small mitochondria which show an irregularity in size and internal structure. The Golgi apparatus is composed of a few short stackled lamellae. Although a number of small vesicles are present, the RER is sparse. Ribosomes are mostly in free form. A large nucleolus may be found at the periphery of the nucleus. Approximately $\times 34,000$.

4 A portion of the cytoplasm of a developing fibroblast from the growing end of a guinea pigs incisor pulp. In comparison with figure 3 a more extensive RER may be seen. The number of ribosomes might also be greater. The irregularity in the structure of mitochondrial cristae is similar to that found in figure 3. Approximately $\times 42,000$. 


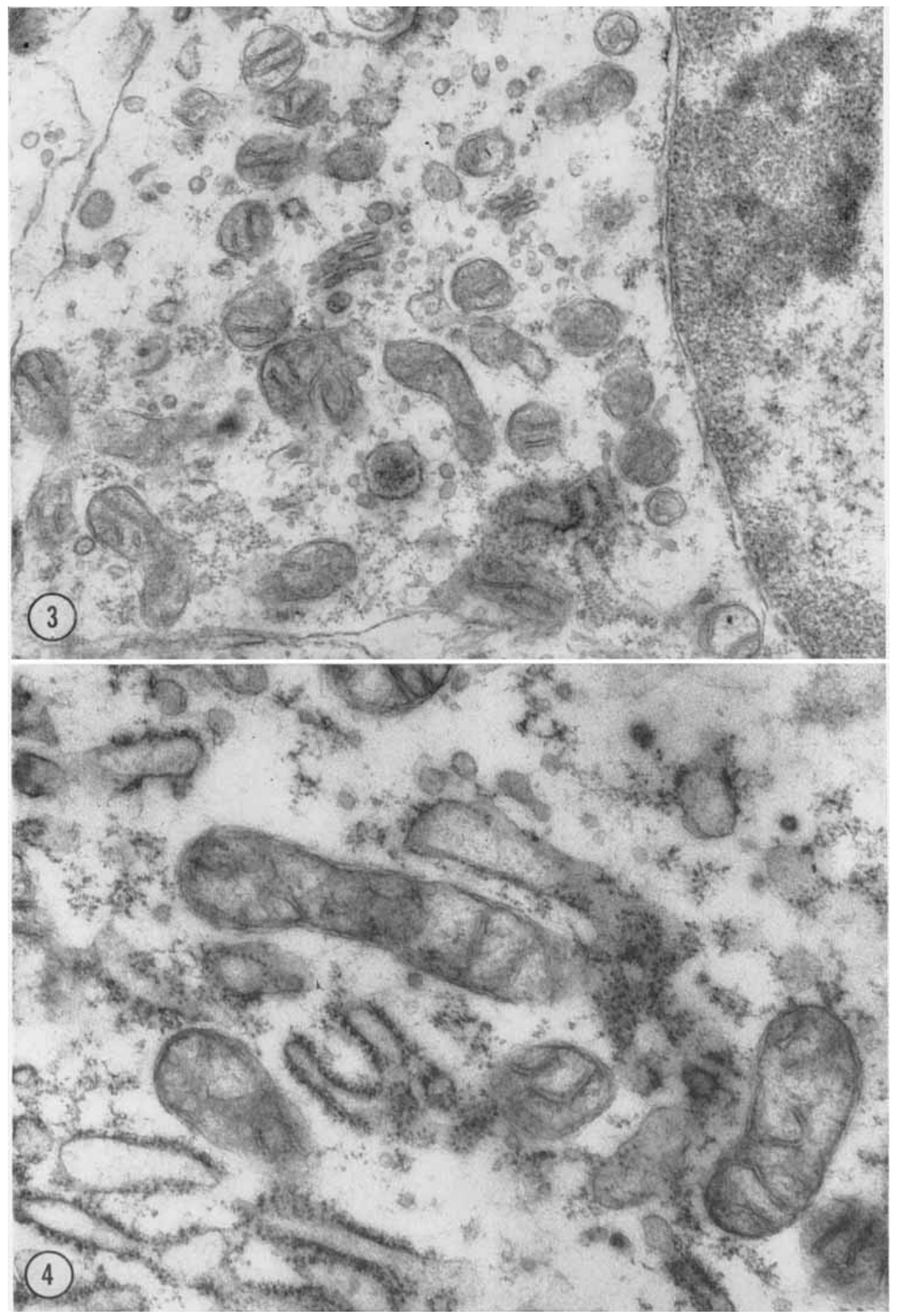


PLATE 3

EXPLANATION OF FIGURE

5 A portion of the cytoplasm of a fibroblast from the midportion of a guinea pig incisor pulp. Numerous mitochondria, when sectioned longitudinally, show well-developed straight cristae. The RER is fairly extensive and contains electron-dense materials within the cisternae. In the region of cytocentrum a portion of cilium is shown, being halfway surrounded by extracellular space produced by the invagination of plasma membrane (arrow). The increased irregularity of the nuclear membrane is apparent. Approximately $\times 32,000$. 


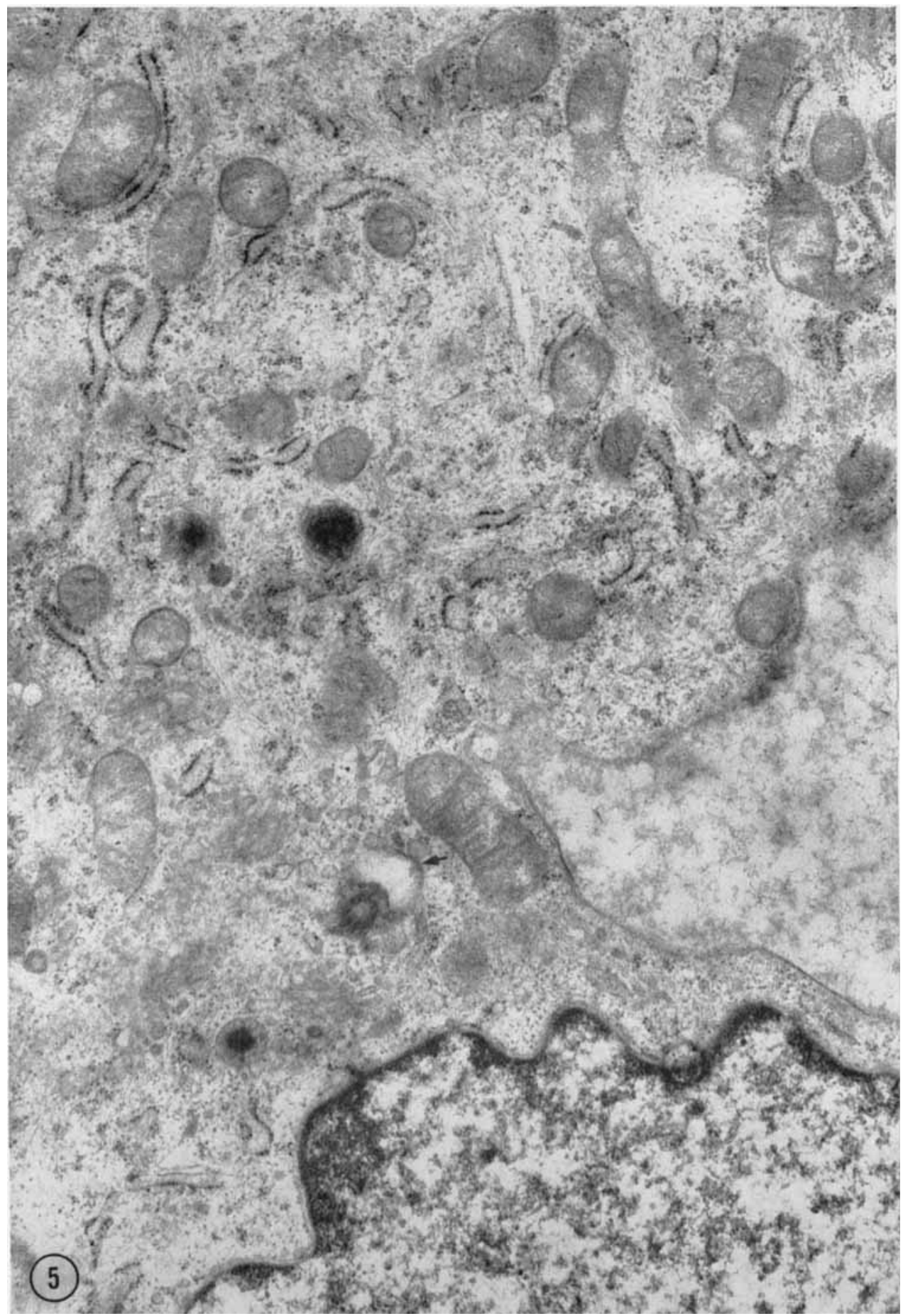


PLATE 4

EXPLANATION OF FIGURE

6 A region of the Golgi complex of a fibroblast from the midportion of a guinea pig incisor pulp. The extensiveness of the lamellar component is well demonstrated. The continuity of the vesicular element with the RER as well as with the lamellar elements may be seen (arrows). Fibrillar materials are enclosed by smooth membranes. Approximately $\times 53,600$. 


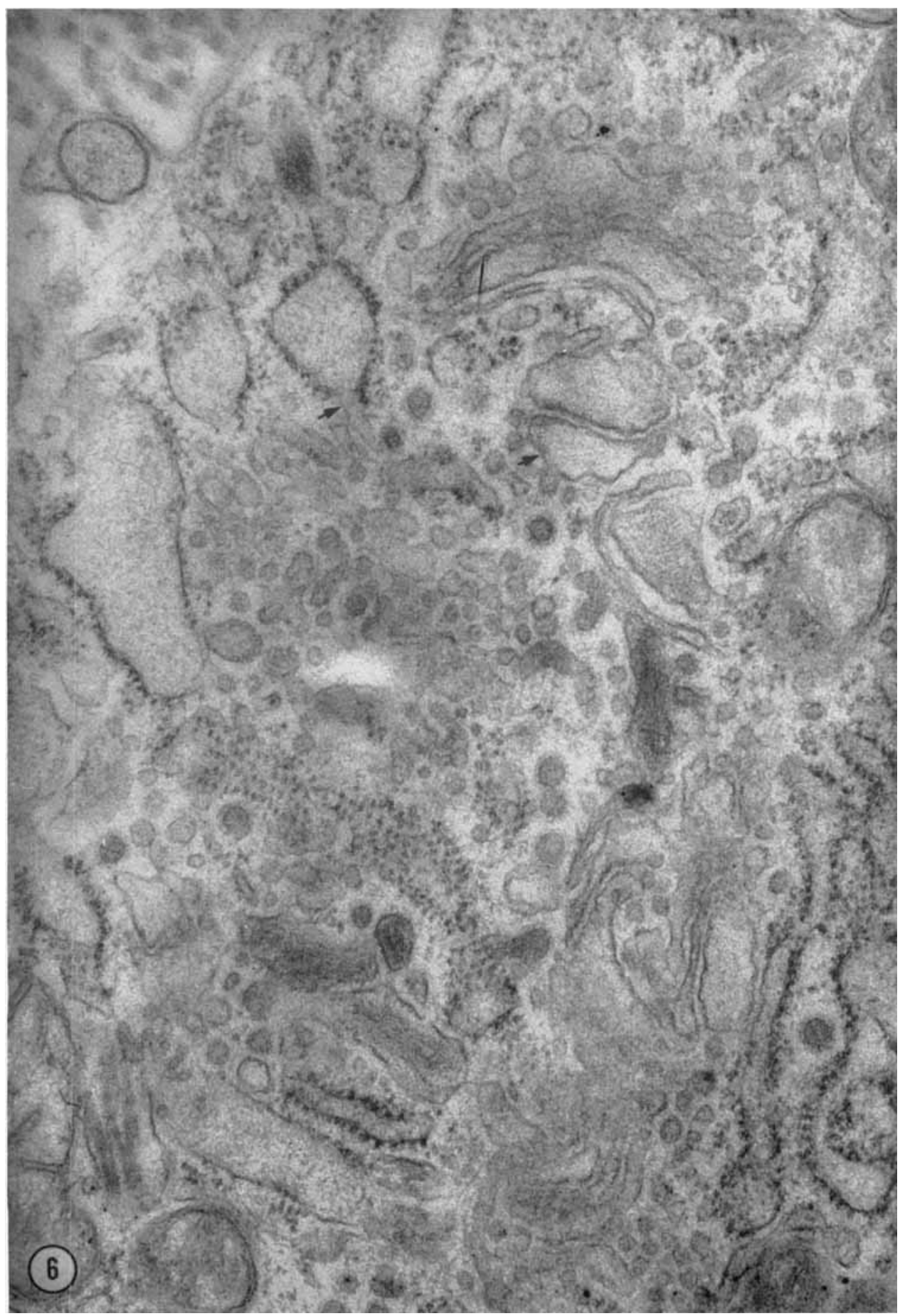


PLATE 5

EXPLANATION OF FIGURES

7 A portion of the peripheral cytoplasm of a fibroblast from the midportion of a guinea pig incisor pulp. Within the irregular-contoured cytoplasm are located vacuoles, well-developed RER, dense bodies and mitochondria. Materials in the cisternae of the flat RER are much more electrondense than the rest of the cytoplasm and extracellular space. Approximately $\times 15,600$.

8 A portion of the cytoplasm of a fibroblast from the midportion of a guinea pig incisor pulp. The RER is dilated to a great degree, and contains somewhat floccular materials which have an electron density about equal to that of the extracellular materials. Approximately $\times 18,000$. 

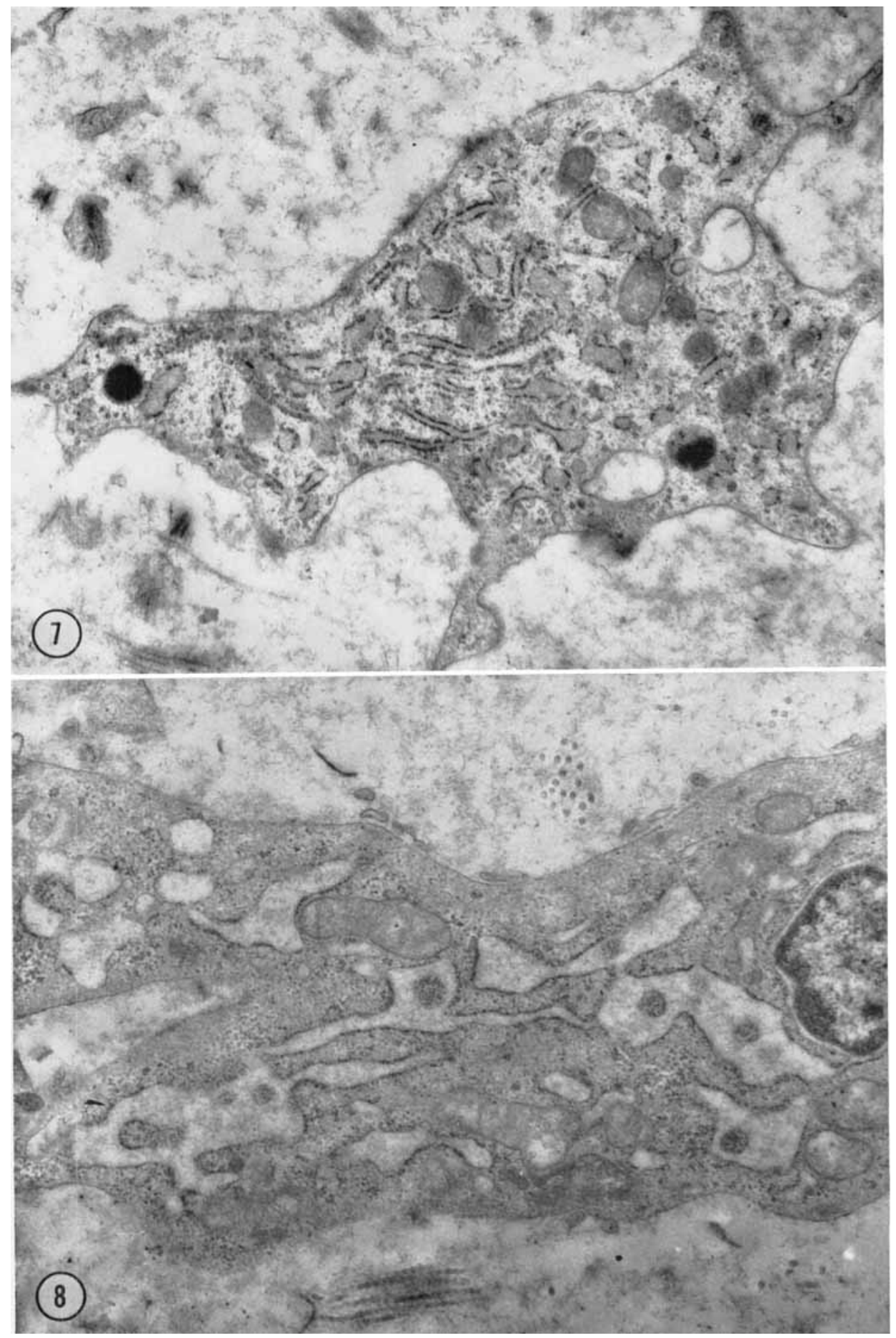
PLATE 6

EXPLANATION OF FIGURES

Portions of the peripheral cytoplasm of the fibroblast from the midportion of a guinea pig incisor pulp

9 The surface membrane shows an invagination which appears to form small vesicles. A heavy tract of the fine fibrils is present under the plasma membrane. Approximately $\times 34,600$.

10 Pieces of cytoplasm located near the cell surface. Their appearance suggests that the adjacent cell might be liberating chunks of the cytoplasm. Approximately $\times 40,000$.

11 A portion similar to that in figure 10. In this case, however, a vacuole is found within the piece of cytoplasm which appears to be in the process of pinching off. The vacuolar structure within the cytoplasm resembles the RER elsewhere. Approximately $\times 32,000$.

12-13 Dense granules enclosed in membranes are located close to the surface plasma membrane. Approximately $\times 39,600$. 

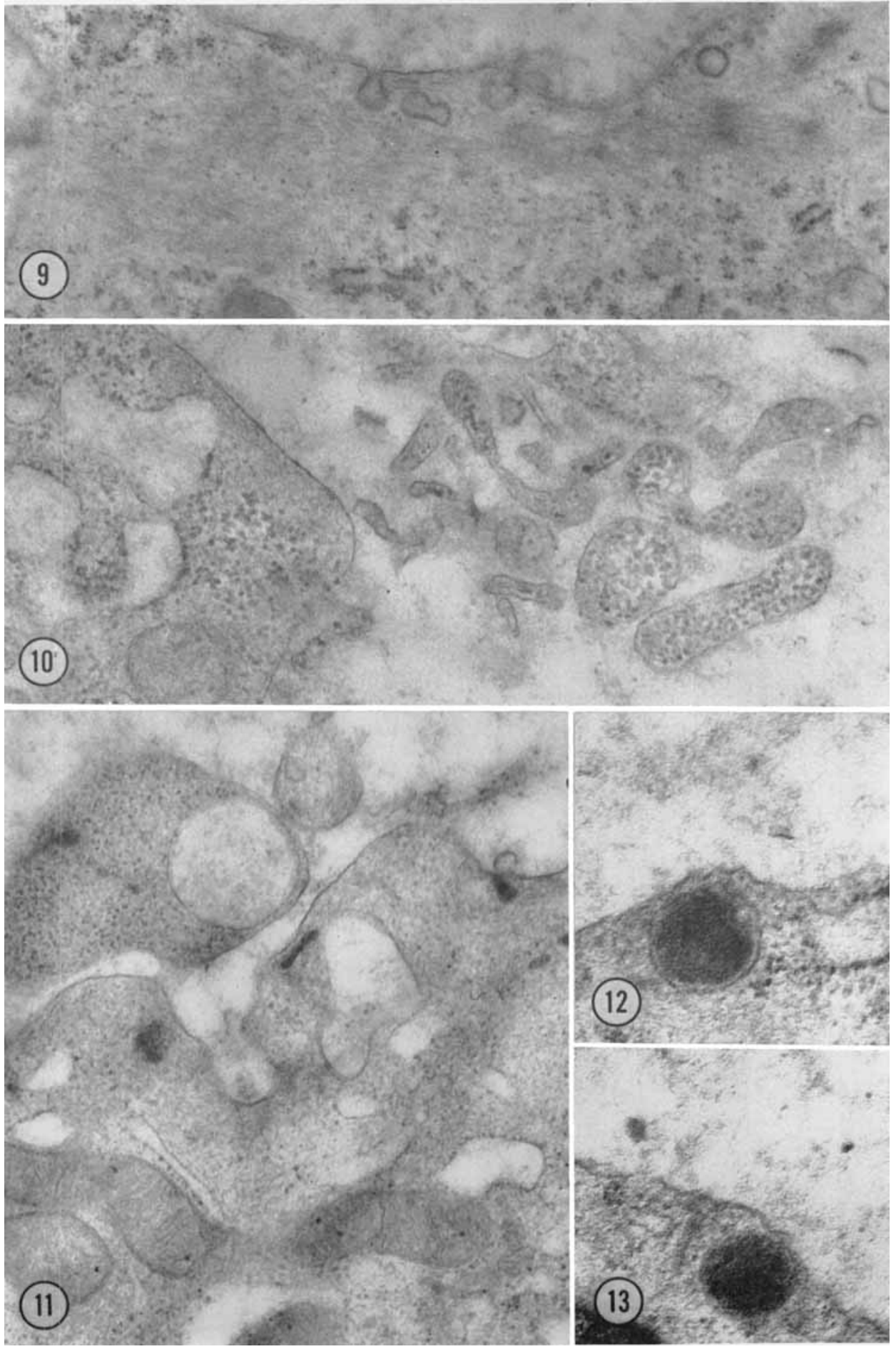
PLATE 7

EXPLANATION OF FIGURES

14 A portion of the cytoplasm of a fibroblast from the apical third of a guinea pig incisor pulp. Presence of dilated RER and well-developed mitochondria resembles the cells found in the middle third of the pulp. However, the number of fine intracellular fibrils appears to have increased. Approximately $\times 34,000$.

15 A fibrocyte from the apical third of a guinea pig incisor pulp. The amount of perinuclear cytoplasm is small, and most cytoplasmic organelles show a decrease in size as well as in the degree or organization. Approximately $\times 16,000$. 


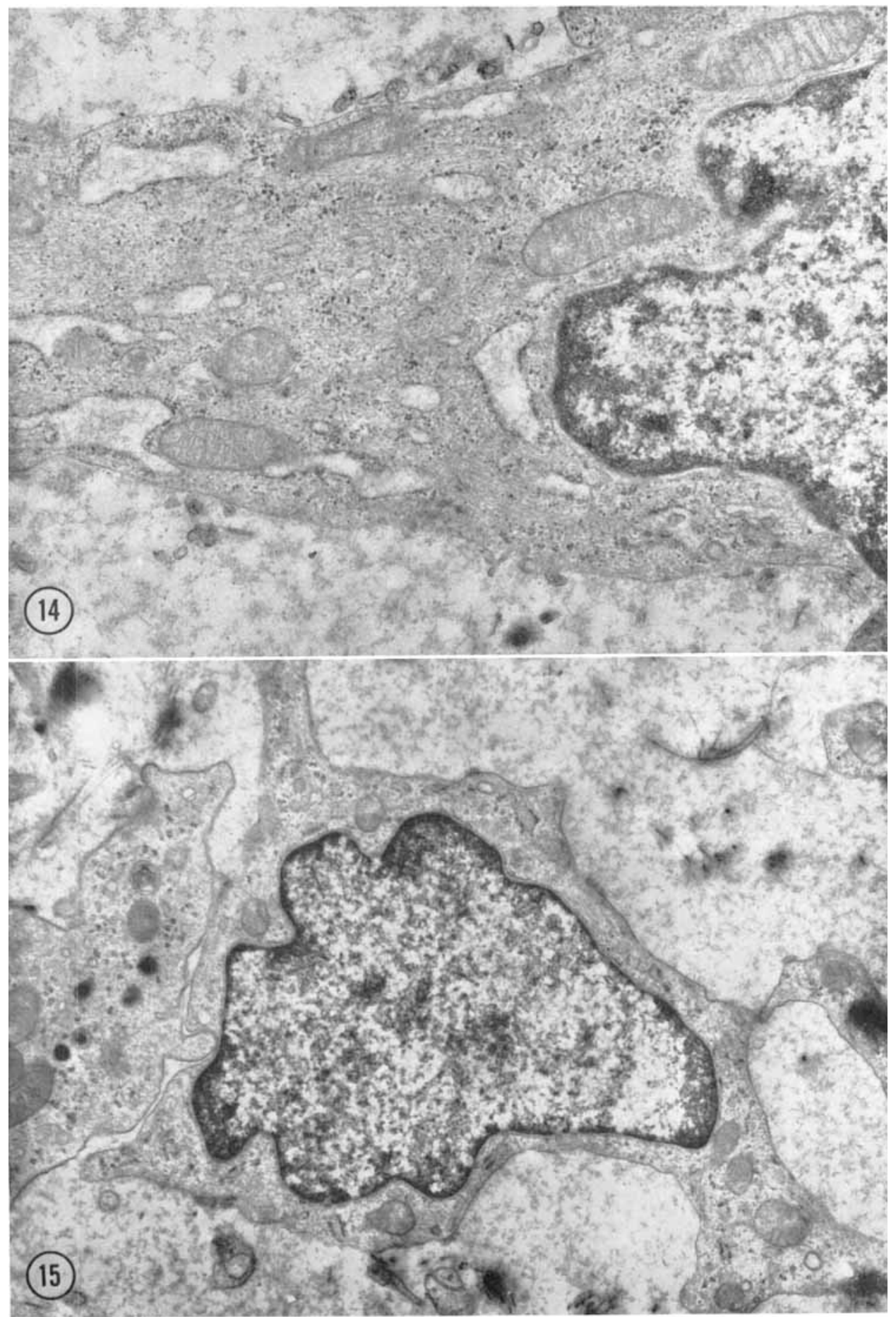


PLATE 8

EXPLANATION OF FIGURES

16 Portions of the peripheral cytoplasm of fibrocytes from the apical third of a guinea pig incisor pulp. Few cytoplasmic organelles are seen except a small number of vesicles and ribosomal clusters. The intercellular fibrils are numerous. Extracellularly many collagen fibrils may be recognized. Approximately $\times 36,000$.

17-18 Appearances of cilia in fibroblasts from the middle third of a guinea pig incisor pulp. The fine structure of cilia confirms observations made of other cell types. Approximately $\times 32,000$. 

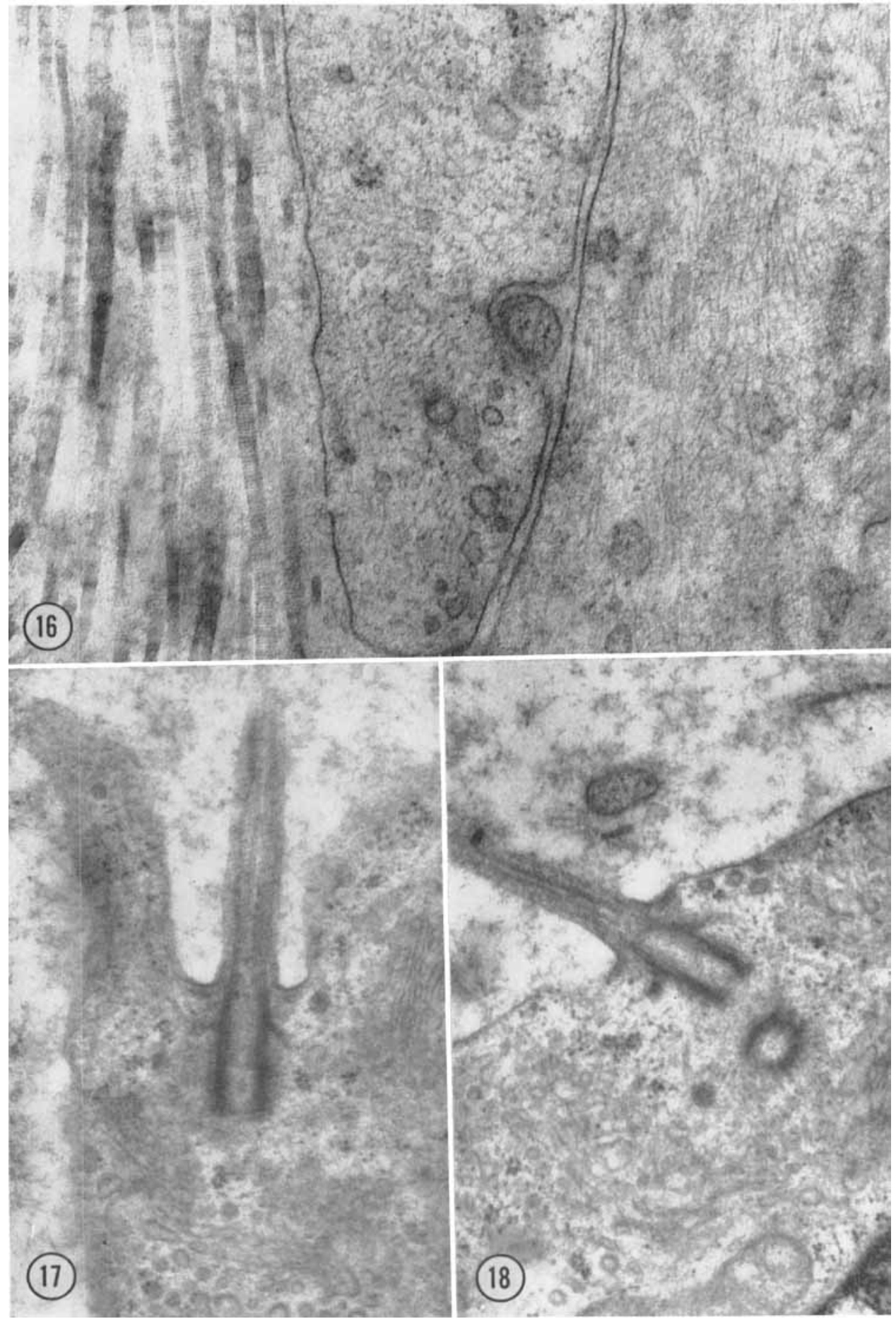